\title{
Modelagem in silico das propriedades farmacocinéticas de ligantes com atividade antitumoral para Sarcoma 180
}

\author{
Lorrana Maíssa Silva do Nascimento ${ }^{1,2}$, Teresinha de Jesus Aguiar dos Santos \\ Andrade $^{2,3}$, Joaquim Soares da Costa Júnior ${ }^{3}$, Danielle da Costa Silva ${ }^{3}$ \\ ${ }^{1}$ Curso de Licenciatura em Ciências Biológicas - Instituto Federal do Maranhão (IFMA) \\ Campus Timon, Timon, MA - Brasil \\ ${ }^{2}$ Núcleo de Investigação Aplicado a Ciências - Instituto Federal do Maranhão - Campus \\ Presidente Dutra, Presidente Dutra, MA - Brasil \\ ${ }^{3}$ Laboratório de Química Orgânica de Produtos Naturais - Instituto Federal do Piauí \\ (IFPI) - Campus Teresina Central, - Teresina, PI - Brasil \\ lorranamaissa141@gmail.com, teresinha.andrade@ifma.edu.br, \\ jquimjr@gmail.com, dcsdanielle@gmail.com
}

\begin{abstract}
Sarcoma 180 (S180) is a type of breast cancer, which is associated with high morbidity and mortality and, therefore, the elucidation of molecular mechanisms is important. The advancement of Medicinal Chemistry in the planning of drugs in silico has been arousing the interest of scientists and pharmaceutical industries. Thus, this work has as main objective to analyze pharmacokinetics parameters (ADME) in silico, using the PreADMET software, for ligands derived from ring $A$ eight pyrrole, substituted by duocarmycin B2, which antitumor evaluation in 5180 . The results obtained corroborate with molecular information that is essential to predict the oral bioavailability of potent drug candidates.
\end{abstract}

Resumo. O Sarcoma 180 (S180) é um tipo de câncer de mama, que está associado à uma alta morbimortalidade e, por isso, a elucidação dos mecanismos moleculares é importante. O avanço da Química Medicinal no planejamento in silico de fármacos e vem despertando o interesse de cientistas e indústrias farmacêuticas. Assim, este trabalho tem como principal objetivo avaliar parâmetros farmacocinéticos (ADME) in silico, por meio do software PreADMET, para ligantes derivados de pirrole de anel A oito, substituídos de duocarmicina B2, com avaliação antitumoral em $S 180$. Os resultados inéditos obtidos corroboram com informações moleculares que são essenciais para prever a biodisponibilidade oral dos potentes candidatos à fármacos.

\section{Introdução}

A Química Medicinal (QM) estuda as complexas relações entre as propriedades físicoquímicas e as interações espaciais da estrutura química de uma molécula com sua atividade biológica, bem como sua interação pelo receptor-alvo no organismo [Serafini et al. 2020]. Estudos in silico é uma das técnicas mais exploradas na Química Medicinal para descoberta de novos medicamentos, usando uma ampla variedade de métodos computacionais [Veljkovic 2019], servindo de complemento aos estudos in vivo e in 
vitro, além da rapidez de sua execução, baixo custo e redução da utilização de animais em ensaios [Macedo et al. 2013]. São baseados no conhecimento da estrutura do receptor alvo (à base do alvo) ou na estrutura química de pequenas moléculas ativas (à base de ligante) e, são rotineiramente usados para descobrir e otimizar o sucesso de compostos de interesse farmacêutico, sendo esta uma área da Química que tem despertado grande propensões dos cientistas e empresas de biotecnologia [Singla 2015].

Pesquisas envolvendo a descoberta de potentes candidatos à fármacos com atividades antitumorais, em especial, voltadas para o câncer de mama, são importantes, pois esta doença possui um crescimento significativo entre as mulheres brasileiras. Desta forma, este trabalho tem como principal objetivo apresentar estudos in silico ADME de ligantes com atividade antitumoral em S180, associado ao câncer de mama.

\section{Referencial Teórico}

A literatura científica relata que o câncer é a segunda principal causa de mortes no mundo, responsável por cerca de 8,8 milhões de óbitos em 2018 [Bastos 2011; WHO 2017]. É considerada uma doença do material genético, decorrente do acúmulo progressivo de mutações e caracteriza-se pela excessiva e desordenada proliferação celular, podendo adquirir a capacidade de migrar do local de origem para outros órgãos (metástase) [Bastos 2011]. O tipo de câncer mais comum entre as mulheres é o de mama, com prevalência de $29,7 \%$. Sua etiologia é desconhecida, mas sabe-se que é formado pelo desenvolvimento de células malignas, originadas nas glândulas mamárias ou no epitélio ductal, por fatores, genéticos, ambientais e/ou adquiridos [Oliveira Filho 2020]. Dentre os tipos de câncer de mama, têm-se o S180, também denominado tumor de Crocker, uma neoplasia maligna rara, caracterizada por se desenvolver em tecidos mesenquimais e, está associado à alta morbimortalidade [Post 2012]. Ensaios in vivo mostram que este tipo de tumor cresce rapidamente, em torno de $90 \%$ a $100 \%$ dos casos [Ferreira et al. 2019] e, por isso, foi selecionado para este estudo.

Sabe-se que é de suma importância descobrir o tumor e tratá-lo precocemente, para aumentar a sobrevida dos pacientes e o avanço da ciência e da tecnologia trouxe profundos benefícios para a área medicinal, dada a interdisciplinaridade envolvendo inúmeras áreas do conhecimento, tornando-a mais ampla e possibilitando uma maior compreensão das razões moleculares da ação dos fármacos, da relação entre as estruturas dos compostos e a atividade farmacológica, tendo em vista fatores farmacodinâmicos e farmacocinéticos (ADMET), avaliando propriedades de absorção, distribuição e excreção, que se constituem em propriedades farmacoterapeuticamente úteis [Viegas Jr et al. 2006], daí a relevância desta pesquisa, envolvendo técnicas de inteligência artificial (IA), com base no planejamento de fármacos auxiliado por computador (“Computer-Assisted Drug Design”- CADD).

\section{Materiais e Métodos}

\subsection{Conjunto de Dados - Data Set}

A princípio, foram realizadas buscas minuciosas na literatura científica, de moléculas sintetizadas em laboratório e, que apresentassem avaliação antitumoral em S180, para compor o conjunto de dados (data set). Os levantamentos realizados evidenciaram a existência de poucos compostos com atividade antitumoral em S180. Assim, os ligantes 
selecionados foram sintetizados por Nagamura et al. [1996], sendo formado por 6 compostos, os quais são derivados de pirrole de anel A oito, e substituídos de duocarmicina B2, que foram enumerados de $\mathbf{1}$ à $\mathbf{6}$ e são apresentados na Figura 1.

Figura 1. Conjunto de dados [Nagamura et al. 1996]

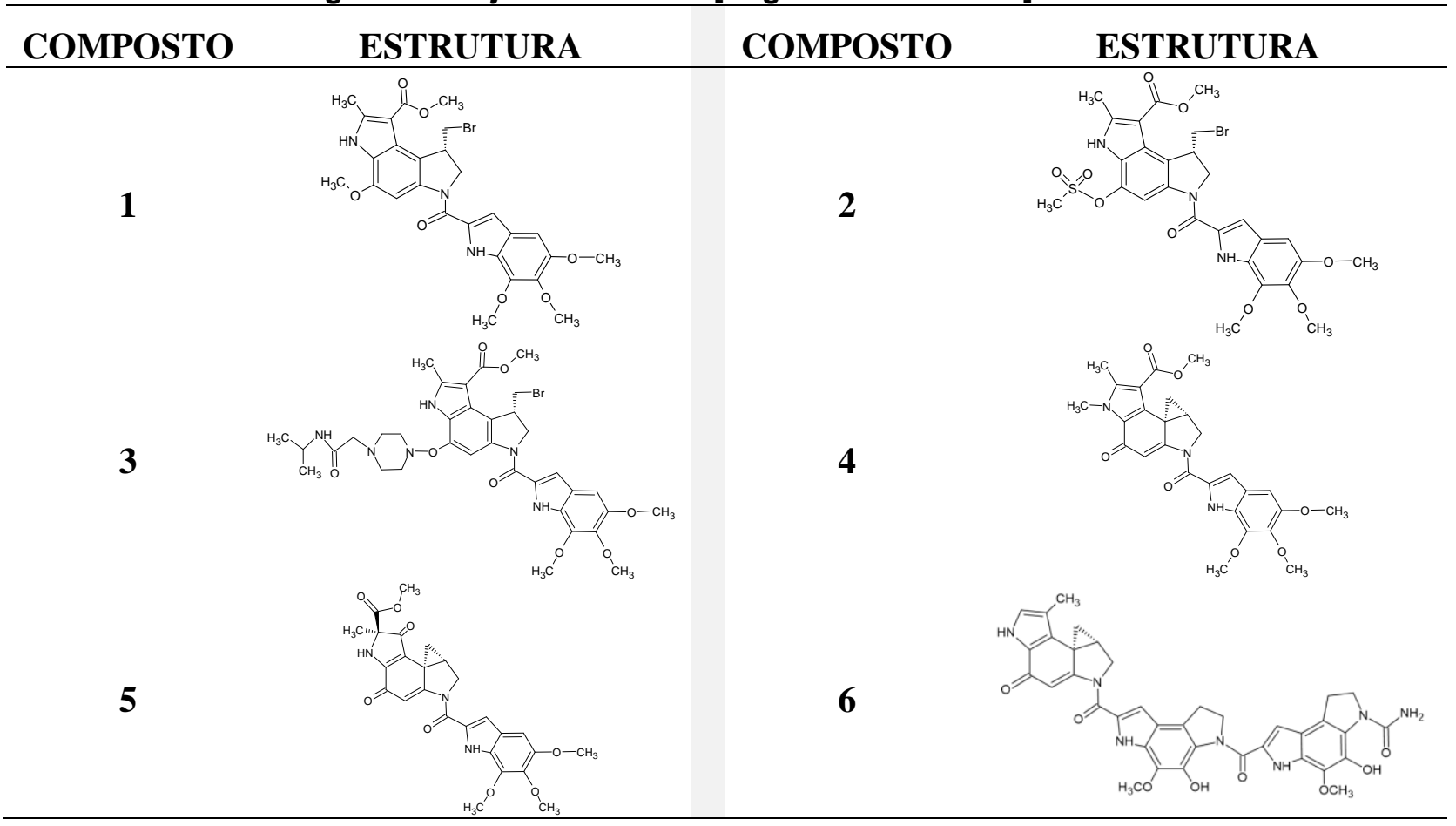

\subsection{Métodos Computacionais - Modelagem in silico ADME}

As estruturas químicas foram desenhadas utilizando o software freeware ChemSketch ACDLabs (versão 10.04, 2008). A estrutura de cada molécula foi conferida para que não houvesse erros nas análises e, consequentemente, no resultado da pesquisa.

A etapa seguinte, foi a análise preditiva dos parâmetros farmacocinéticos do data set e, para isto, utilizou-se o web servidor PreADMET (versão 2.0, Copyright@ 20052017, $B M D R C)$, que analisa parâmetros de absorção, distribuição e excreção de compostos, além dos descritores de metabolismo e toxicidade e, detém a finalidade de demonstrar resultados preliminares in silico importantes para o processo de descoberta de novos fármacos [Sousa et al. 2020], por meio de cálculos seguindo a regra de Lipinski, em que uma molécula deve apresentar valores para 4 parâmetros múltiplos de 5, sendo eles: $\log \mathrm{P}$ menor ou igual a 5, com massa molecular menor ou igual a 500, aspectos ligantes de Hidrogênio menor ou igual a 10, além de doadores de ligação de Hidrogênio menor ou igual a 5 [Dolabela et al. 2018], também se baseia na relação estrutura atividade (SAR) das moléculas a serem analisadas com outras previamente pesquisadas, reunindo informações presentes em diversos bancos de dados, para predizer resultados importantes no processo de triagem de moléculas candidatas a fármacos. Este software é mantido pela Universidade de Seul, na Coreia do Sul e, encontra-se disponível em: https://preadmet.bmdrc.kr/ [Bastos 2020].

\section{Resultados e Discussão}




\subsection{Caracterização Biológica do Conjunto de Dados - Data Set}

As análises obtidas neste estudo foram realizadas a partir de um conjunto de dados, formado por moléculas que apresentaram atividade antitumoral em Sarcoma 180, nos ensaios in vitro, descritos na literatura científica selecionada [Nagamura et al. 1996].

Estes compostos são derivados pirrole de anel A oito, substituídos de duocarmicina B2. A duocarmicina B2 é um tipo de antibiótico antitumoral isolado do caldo de cultura de Streptomyces $s p$ e as sínteses realizadas foram feitas a partir do anel 2-metil-3-metoxicarbonil-Apirrol-DUMA, presentes na estrutura. A região do carbono fenólico foi utilizada para formar os derivados substituintes, dentre os quais têm-se: hidroxilas, éter, sulfonato, éster, carbonato, carbamato e cianos [Nagamura et al. 1996].

\subsection{Análises dos Parâmetros Farmacocinéticos (ADME) in silico}

Os seguintes parâmetros de absorção e excreção foram analisados: passagem da barreira hematoencefálica (BBB); permeabilidade em Caco2 (Caco2); absorção no intestino humano (HIA); permeabilidade em MDCK (MDCK); ligação às proteínas plasmáticas (PPB); permeabilidade na pele (SP) e inibição da glicoproteína P (PGP inibição). A Tabela 1 mostra os resultados obtidos para as propriedades farmacocinéticas analisadas.

Tabela 1. Parâmetros de absorção e excreção

\begin{tabular}{cccccccc}
\hline COMPOSTO & BBB & Caco2 & HIA & MDCK & PPB & SP & PGP inibição \\
\hline $\mathbf{1}$ & 0,832 & 22,381 & 92,625 & 0,041 & 87,431 & $-4,461$ & Sim \\
$\mathbf{2}$ & 0,150 & 15,935 & 93,255 & 0,042 & 94,083 & $-2,708$ & Sim \\
$\mathbf{3}$ & 0,230 & 22,057 & 92,420 & 0,043 & 62,263 & $-4,719$ & Sim \\
$\mathbf{4}$ & 0,045 & 29,246 & 94,341 & 0,043 & 72,151 & $-4,726$ & Sim \\
$\mathbf{5}$ & 0,062 & 7,541 & 91,285 & 0,046 & 44,121 & $-4,947$ & Não \\
$\mathbf{6}$ & 0,078 & 13,705 & 76,233 & 0,043 & 80,728 & $-5,278$ & Sim \\
\hline
\end{tabular}

Legenda: BBB - barreira hematoencefálica; Caco2 - permeabilidade em Caco2; HIA absorção no intestino humano; MDCK - permeabilidade em MDCK; PPB - ligação às proteínas plasmáticas; SP - permeabilidade na pele, PGP inibição - inibição da glicoproteína P.

A análise da Tabela 1 apresenta uma propriedade importante à respeito da capacidade de uma droga penetrar na BBB [PreADMET 2017]. Os resultados mostram que os compostos 4,5 e 6 possuem uma baixa absorção $(<0,1)$ e os demais classificamse como média absorção $(0,2$ à 0,1$)$. Essa análise limita e restringe a passagem de substâncias da corrente sanguínea para o cérebro devido sua alta impenetrabilidade e seletividade [Bastos et al. 2020].

Já a permeabilidade em células Caco2, permite prever a capacidade de absorção in vivo, fundamental em estudos pré-clínicos. Estas células são oriundas de adenocarcinoma de cólon humano e possuem a capacidade de se diferenciarem em enterócitos, absorvendo e transportando moléculas para diversas partes do corpo [Dolabela et al. 2018]. Todos os compostos analisados apresentam valores que variam de 7 a 30 para este parâmetro, sendo classificados como permeabilidade média em células Caco2 (4 a 70). Isso indica que fármacos oriundos dessas estruturas químicas podem não ter absorção completa para que sejam distribuídos no organismo. Além das células Caco2, as MDCKs também são utilizadas para prever a absorção de compostos, 
pois ambas ao serem cultivadas em membranas semipermeáveis diferenciam-se em células epiteliais colunares e apresentam junções semelhantes. Os compostos analisados possuem baixa permeabilidade em células MDCK, uma vez que apresentam valores $<4,0$ para este descritor [Dolabela et al. 2018; Sousa et al. 2020].

O parâmetro de HIA, um dos mais importantes para o perfil de ação de fármacos administrados por via oral tendo como foco a absorção no trato gastrointestinal (TGI) [Ando, Hisaka and Suzuki 2015]. Todos os compostos avaliados apresentaram taxas acima de $76 \%$ para o HIA, indicando uma alta absorção (de 70\% a 100\%). Frisa-se que o valor ideal da HIA de um fármaco dependerá de sua finalidade farmacêutica e, deve ser produzido de acordo com a necessidade da doença estudada [Dolabela et al. 2018].

A PPB é uma propriedade importantíssima no planejamento e desenvolvimento de novos fármacos, pois quanto maior o número de moléculas $\mathrm{PPB}$, maior sua capacidade de atuar como forma de reservatório, aumentando a disponibilidade do fármaco no organismo. E aquelas que não se ligam à essas proteínas podem apresentar maior difusão para os tecidos e, consequentemente, sua taxa de eliminação será maior [Santos et al. 2018]. Assim, ao analisar os dados para este descritor, apenas o composto 2 possui valor acima de $90 \%$, indicando uma substância com PPB forte; já os demais compostos apresentaram percentual de $44 \%$ à $88 \%$, o que evidencia uma PPB fraca.

A SP é um fator significativo ao tratar-se de substâncias que serão administradas por via transdérmica, para verificar a existência de perigo dos compostos ao ter contato com a pele [Bastos et al. 2020] e as análises evidenciam que todos os compostos possuem valores $<0,1$ para este descritor, indicando uma alta SP. Por fim, a análise da PGP inibição, encontrada, sobretudo em células epiteliais encarregadas de revestir o intestino delgado, hepatócitos, colón e glândula renal, que possui função excretora, auxiliando na expulsão de toxinas e xenobióticos dos tecidos [Sousa et al. 2020], evidencia que, apenas o composto 5 não inibe a PGP e, isso acarreta numa redução da sua biodisponibilidade.

\section{Conclusão e Trabalhos Futuros}

Os resultados obtidos indicam que os ligantes estudados possuem propriedades ADME importantes, que contribuem para pesquisas voltadas no planejamento e desenvolvimento de fármacos com mecanismos antitumorais associados ao S180. Vale ressaltar que análises metabólicas in silico dos ligantes usados neste estudo, estão em andamento e a predição de estudos de toxicidade será realizada na etapa seguinte.

\section{Agradecimentos}

Os autores agradecem a Fundação de Amparo a Pesquisa do Estado do Maranhão (FAPEMA), Fundação de Amparo a Pesquisa do Estado do Piauí (FAPEPI), Instituto Federal do Maranhão (IFMA), Instituto Federal do Piauí (IFPI) e ao Conselho Nacional de Desenvolvimento Científico e Tecnológico (CNPq).

\section{Referências}

Ando, H.; Hisaka, A. and Suzuki, H. (2015) "A new physiologically based pharmacokinetic model for the prediction of gastrointestinal drug absorption: translocation model". Drug Metabolism and Disposition, v. 43, n. 4, p. 590-602. 
Bastos, I. V. G. A. (2011) "Avaliação da atividade farmacológica de Caesalpinia echinata Lam. (Flores)". Dissertação de Mestrado. Universidade Federal do Pernambuco.

Bastos, K. Z. C. et al. (2020) “Análise in silico do perfil farmacocinético e toxicológico de fármacos em pesquisa para o tratamento da COVID-19". Pesquisa, Sociedade e Desenvolvimento, v. 9, n. 11, p. e529119450-e529119450.

Dolabela, M. F. et al. (2018) "Estudo in silico das atividades de triterpenos e iridoides isolados de Himatanthus articulatus (Vahl) Woodson". Fitos, v.12, p. 227-242.

Ferreira, P. M. P. et al. (2019) Biologia e aplicações pré-clínicas do modelo experimental sarcoma 180. In: OLIVEIRA JUNIOR, José Max Barbosa. Análise crítica das ciências biológicas e da natureza 2. Ponta Grossa: Atena Edit., p. 270-287.

Nagamura, S. et al. (1996) "Synthesis and antitumor activity of duocarmycin derivatives: A-ring pyrrole analogues of duocarmycin B2". Bioorganic \& Medicinal Chemistry, v. 4, n. 8, p. 1379-1391.

Macedo, K. G.; Melo Filho, C. C.; Andrade, C. H. (2013) "Avaliação in silico da toxicidade de fármacos”. Revista Eletronica de Farnácia, v. 10, n. 1, p. 1-1.

Oliveira Filho, J. W. G. (2020) “Avaliação toxicogenética de produtos naturais isolados de fungos associados de algas marinhas para formulações antitumorais". Tese de Doutorado em Biotecnologia, RENORBIO, Universidade Federal do Piauí.

Post, S. M. (2012) "Mouse models of sarcomas: critical tools in our understanding of the pathobiology". Clinical sarcoma research, v. 2, n. 1, p. 1-9.

PreADMET. Predição ADME, (2017). https://preadmet.bmdrc.kr/adme-prediction/. Acesso em: Fevereiro de 2021.

Santos, R. C. et al. (2018) "Modificação molecular incremental: análise de parâmetros físico-químicos, farmacocinéticos e toxicológicos in silico de fármacos inibidores seletivos da recaptação de serotonina (ISRSs)”. Boletim Inf. Geum, v. 9, n. 2, p. 31.

Serafini, M. et al. (2020) "Essential Medicinal Chemistry of Essential Medicines". Journal of medicinal chemistry, v. 63, n. 18, p. 10170-10187.

Singla, R. K. (2015) "In silico drug design \& medicinal chemistry". Current topics in medicinal chemistry, v. 15, n. 11, p. 971.

Sousa, G. A. et al. (2020) "Análise in silico da farmacodinâmica, farmacocinética e toxicidade de dois compostos isolados da Actinidia deliciosa para investigação do seu potencial anti-hiperlipêmico". Research, Society and Development, v. 9, n. 7, p. e790974679-e790974679.

Veljkovic, N. (2019) "Recent in silico resources for drug design and discovery". Current medicinal chemistry, v. 26, n. 21, p. 3836-3837.

Viegas Jr., C.; Bolzani, V. S.; Barreiro, E. J. (2006) "Os produtos naturais e a química medicinal moderna". Química Nova, v. 29, n. 2, p. 326-337.

WHO. World Health Organization. (2017) Câncer. Disponível em: http://www.who.int/cancer/en/index.html. Data de acesso: Dezembro de 2020. 